\title{
Wavelet based analysis of truck vibrations during off-road transportation
}

\author{
Martin Vlkovský1 ${ }^{1}$ Piotr Koziol ${ }^{2}$, and Dariusz Grzesica ${ }^{2, *}$ \\ ${ }^{1}$ University of Defence, 66210 Kounicova street 65, Czech Republic \\ ${ }^{2}$ Cracow University of Technology, Warszawska 24, 31-155 Krakow, Poland
}

\begin{abstract}
Analysis of vibration of the vehicle on the road surface is of importance for cargo and vehicle security. The vibration data analysed in this paper include vibrations in three directions: longitudinal, transverse and vertical. They have been collected for military cargo during transport on various road surfaces. Compared to previous publications, in this paper, a wavelet transform is proposed for vibration analysis, with the emphasis on their efficiency and the capability to recognise important details of the signal. A series of simulations is needed for making an appropriate chose of wavelets, in order to analyse the most wanted features in time-frequency domain. Wavelet coefficients can be analysed for a wide range of frequencies. The main purpose of this study is to extract from the obtained scalograms essential features allowing to recognize a type of the road for which the signals were recorded. The obtained results might support the identification procedure for the road recognition based on measured signals, especially for vehicles passing from one type of road to another.
\end{abstract}

\section{Introduction}

Cargo securing of trucks and loads is a long-term problem. According to statistics $25 \%$ of accidents involving trucks are caused by inadequate cargo securing in the road transport. It is generally affected by three main factors: road infrastructure, truck and the driver [1]. The paper deals with first factor, which can be simulated or modelled based on real-measured data. Second factor, vehicle, is very often solved aspect of the transportation when the simulation software (for instance MSC.ADAMS) is used. Within this simulation the dynamics of moving parts is studied, e.g. in [2] or [3]. Third factor, driver or better said driving style is a problem mostly for traffic psychologists. However, all these aspects should be analysed together, including also other factors related to e.g. weather or appearance of unexpected conditions. Therefore they lead, as usually dynamic systems do, to stochastic analysis taking into account random parameters.

The study of the interaction between vehicle and road surface is a mature field of research. One of the first was Vogel [4]. He reveal that the pavement profile spectral estimates were independent of road type, but functions of rms level and roughness. In other hand, according to Dodds and Robson [5], the typical road surface can be considered as a homogeneous and isotropic two-dimensional Gaussian random process that can be

\footnotetext{
* Corresponding author: dgrzesica@pk.edu.pl
} 
described and classified with the use of PSD function. Research made by Heath [6] proved that certain parts of road surface, in some cases, were stationary. The work [7] indicates a need for further investigation in order to provide a more accurate description of relationship between vehicle and road surface.

Recently, there has been growing demand for ensuring the safety of vehicle and cargo during transportation process. Concerning longitudinal, transverse and vertical dynamics the accelerations generated by variations in different kind of road surface induce harmful effects in freight and further impairs of the road surface. In [8] authors provide definitions of shock and vibration environment to which fissile material shipping containers may be exposed during normal shipment by truck and rail cars. The simple analysis of vibration and shocks were presented. Thus the primary task of safe cargo transportation is to provide more information about road recognition by changing from on type of the road to another.

In order to achieve these goals the measurement of accelerations in three dimensions is made. It is proposed that for better recognition of the road surface quality and its physical properties, a wavelet transform will be used for signal analysis. A the first step, a continuous wavelet transform will be used with possibility of scalograms derivation. This should allow to find specific details related to each fragment of the analysed road surfaces. Such expectations are based on previous works of the authors, where short-time acoustic signals are analysed by using complex wavelet function type of Gabor [9]. In this work, continuous wavelet transform results in terms of scalograms analysis were compared with spectrograms obtained by using STFT (short time Fourier transform) leading to interesting results, especially with some intersection of the scalograms.

Various studies published till now include the analysis of relationships between road surfaces and vehicles. Spies [10] invented a method and a device for recognizing the condition of a road. They built a device for detecting the condition of the surface and adjacent edges of a road by means of electromagnetic radiation. In other work, the distributed road surface condition monitoring using mobile phones was developed [11]. The pattern recognition system for detecting road condition from accelerometer and GPS readings was used. There are also works which include specific methods based on accelerometer measurements. In [12] a numerical method to analyse traffic-induced three-dimensional ground vibrations on the basis of a dynamic elastic finite element method was presented. This technique, using an extended thin-layered element method, can account not only for irregularities in near fields, including pavement structures, but also for traveling waves to far fields. Studying the literature the most common method of identification of road surface roughness as an input to vehicle dynamics is the spectral analysis [13].

A range of practical examples illustrate how the wavelet method may be applied to vibration analysis and some of its advantages are presented in $[14,15]$. The new approach of this paper is not only to identify the road surface roughness but to study what is happening when passes from on type of the road to another.

\section{Data measurement}

The measuring was carried out on 28th February 2017 in the Czech Republic. For this purpose, a training polygon (Fig. 1) in the military training area Březina (close to the town of Vyškov) was used. The experiment was conducted on four different types of roads. Blue part of polygon was muddy terrain with several puddles (pools). Green part was gravel and completely dry. Yellow part was covered by cobblestones - the surface was dry as well. The last part, red one, was unpaved with almost dry surface. 


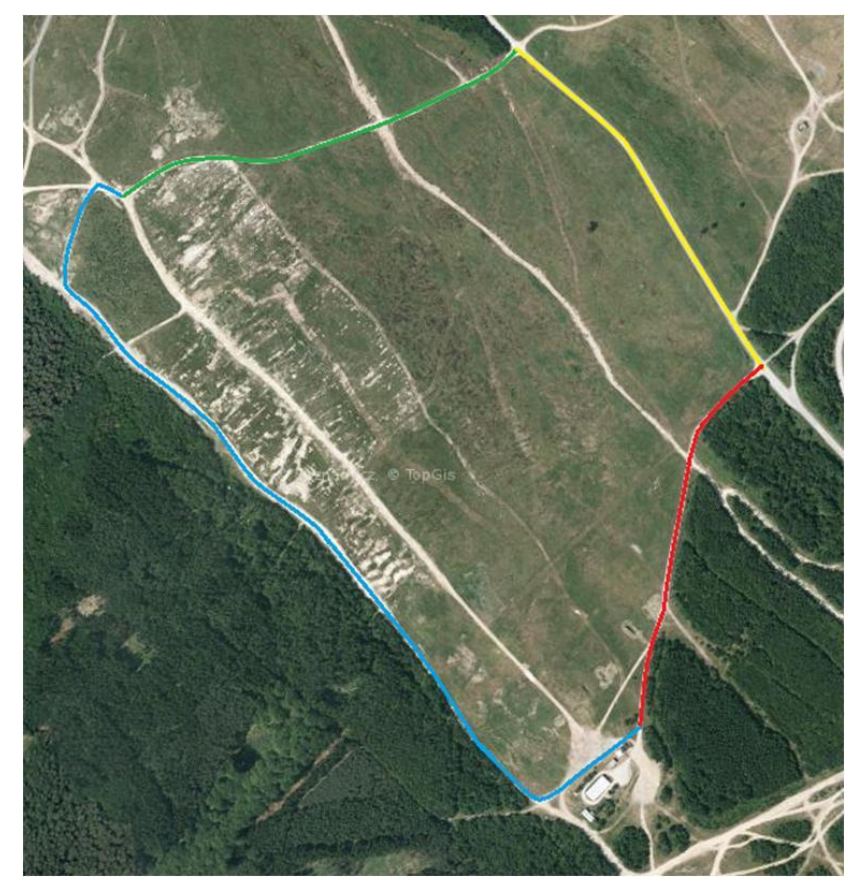

Figure 1. Training polygon with four different kind of road surface

The measurements were taken on a TATRA T-815 truck of VVN 202356 X6.1 R type having effective weight of $8,000 \mathrm{~kg}$ without load. The vehicle was in a good technical condition with the mileage about 150 thousand $\mathrm{km}$. During the transport experiment, there were good climatic conditions, temperature about $3^{\circ} \mathrm{C}$ degree, excellent visibility and no rainfall.

For the measuring, 4 OM-CP-ULTRASHOCK-5-CERT accelerometers with a datalogger, including a calibration certificate, were used. The measuring equipment (accelerometers) measured acceleration (acceleration coefficients) in all axes (X longitudinal, Y - transversal and $\mathrm{Z}$ - vertical). These are dimensionless coefficients specifying the acceleration as a multiple of gravitational acceleration. The highest/lowest values of the acceleration coefficients were recorded every second at the frequency of 512 $\mathrm{Hz}$ for each axis [16].

\section{Wavelet based methodology}

Noise and vibrations generated by transport (vehicles or trains) have usually similar features, i.e. they are characterized by strong variations of several frequency components. However, in some cases, special features allowing characterization of the surface must be sought in more detailed analysis. This is due to the fact that surfaces being compared are almost similar or very close each to another with their characteristics in the transform domain (Fourier analysis), or the surface might be significantly destroyed (or in a very bad condition) and movement of vehicles, especially with high speeds, leads to extremely strong dynamic variations of the measured signals. In the first case, the Fourier tools are not sensitive enough and more exact decomposition of signals in time-frequency domain is needed. In the second case, the Fourier transform, even if it is a windowed modification leading to STFT, lose important details of signals. In both cases, the wavelet techniques give a chance of deeper insights into structure of the signals, mainly to their ability of data 
decomposition at arbitrarily chosen level of time and frequency (i.e. in small time and frequency intervals). This allows denoising of measured signals at any level, depending on computational possibilities only. Another chance might be recognized in structure of some wavelets reflecting fractal character. These wavelets are especially useful when it comes to "digital-zoom" in time-frequency domain.

For the initial study, the complex Gabor wavelet is used, following procedure described in [9]. However, this choice might be improved (optimized) on the basis of employed object identification techniques.

(a)

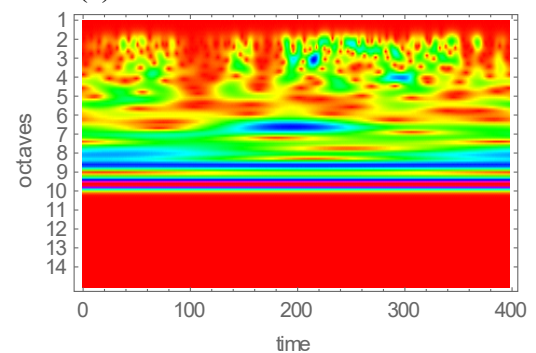

(b)

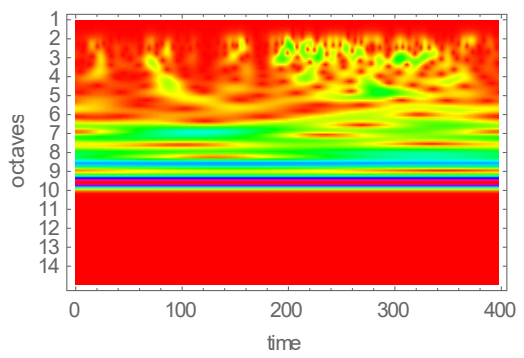

(c)

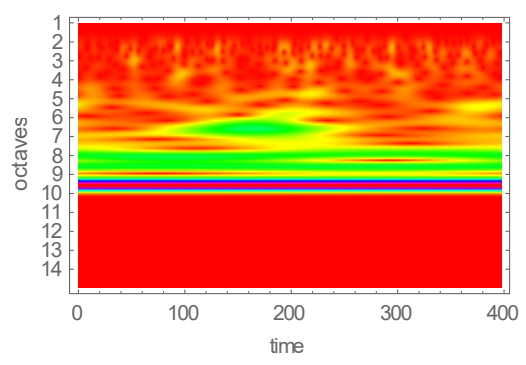

(d)

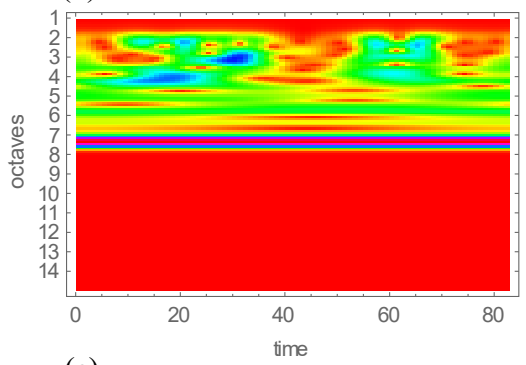

(e)

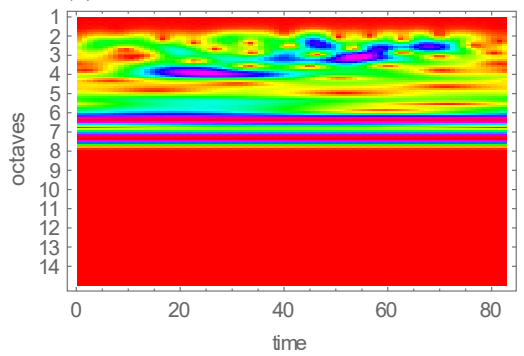

(f)

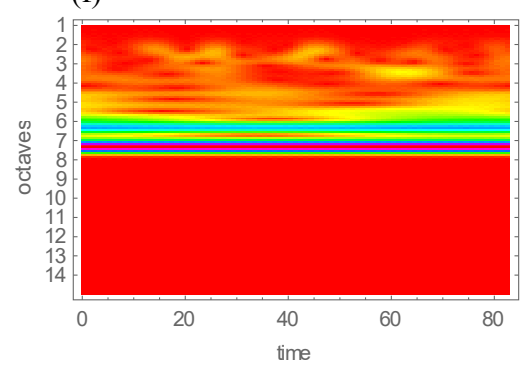

Figure 2. Scalograms of the measured signals transformed by using the continuous wavelet transform with the introduced Gabor wavelet of frequency 6: (a-c) road surface $1-\mathrm{x}, \mathrm{y}, \mathrm{z}$ directions; (d-f) road surface 2 - $\mathrm{x}, \mathrm{y}, \mathrm{z}$ directions.

Figure 2 presents example of the proposed procedure applied to signal recorded on two different road surfaces (the first two described). With only such a simple and preliminary study, one can see significant differences in scalograms characteristics. More detailed analysis is needed for recognition of features allowing the object identification and this is left for future work.

To find appropriate wavelet basis that could give detailed enough description of measured signals in the transform domain, a number of experiments should be performed and extensive comparative studies must be carried out. This will give information about properties of wavelet families enabling the desired analysis. 
The Authors are of the opinion that the signals obtained during measurement described in section 2 are for sure aperiodic, and even might be non-stationary. Therefore techniques based on object identification should be employed to solve the described problem in future.

\section{Conclusions}

The measurements of military truck vibrations during transportation on different road surfaces are collected. The vibration data include vibrations in three directions: longitudinal, transverse and vertical. Because of meaningless results of the Fourier analysis applied to these signals, it is proposed to use instead the wavelet transforms and to find some differentiating factors allowing identification of the road. It is expected that the applied method transferred directly from past analysis of acoustic signal, with some optimization procedure of wavelet filters, can give effective method of the structure identification. This needs, however, further extensive studies and parametrical analysis before formulation of guidelines for application of the developed method.

\section{References}

[1] T. Lerher, Nova Science Publishers, New York (2015)

[2] V. Neumann, Proceedings of Conference. Kaunas, Lithuania: Kaunas University of Technology, 177-180 (2015)

[3] C. Zong, H. Zhang, Z. Yi, Proceedings of 14th COTA International Conference of Transportation Professionals: Safe, Smart, and Sustainable Multimodal Transportation Systems, CICTP 2014 (2014)

[4] W. Vogel, ATZ, 67, 7-11 (1965)

[5] C. J. Dodds, J. D. Robson, JSV, 31, 2, 175-183 (1973)

[6] N. Heath, JSV, 18, 275-284 (1989)

[7] V. Rouillard, Packag Technol Sci. 21, 501-514 (2008)

[8] F. Magnuson, L. T. Wilson, Shock and Vibration Environment for Large Shipping Containers on Rail Cars and Trucks. Sandia Labs., Albuquerque, NM (USA), Report No SAND-76-0427; NUREG-766510 TRN: 77-016345, (1997)

[9] A. Blazejewski, P. Koziol, M. Luczak, Arch Acoust. 39, 3, 385-394 (2014)

$[10]$ H. Spies, W. Weishaupt, Method and a device for recognizing the condition of a road, US Grant US5163319A (1987)

[11] M. Perttunen, O. Mazhelis, F. Cong, M. Kauppila, T. Leppänen, J. Kantola, J. Collin, S. Pirttikangas, J. Haverinen, T. Ristaniemi, J. Riekki, In: Hsu CH., Yang L.T., Ma J., Zhu C. (eds) Proceedings of the International Conference on Ubiquitous Intelligence and Computing, UIC 2011, 6905 (2011)

[12] T. Hanazato, K. Ugai, M. Mori, R. Sakaguchi, J. Geot. Eng. 117(8), 1133-51 (1991)

[13] M. Xu, A. M. O. Mohamed, R. N. Yong, F. Caporuscio, J TERRAMECHANICS, 29(45), 477-486 (1992)

[14]E. Newland, Journal of Vibration and Acoustics, 116(4): 417-425 (1994)

[15] S. Mallat, Academic Press Inc. Ltd, London (1998)

[16] M. Vlkovský, M. Šmerek, J. Michálek, Proceedings of the World Multidisciplinary Civil Engineering-Architecture-Urban Planning Symposium (2017) 